\title{
Data Predistortion for Multicarrier Satellite Channels using Orthogonal Memory Polynomials
}

\author{
Roberto Piazza*, Bhavani Shankar Mysore Rama Rao *, Björn Ottersten*† \\ *Interdisciplinary Centre for Security, Reliability and Trust, (SnT), University of Luxembourg, \\ email: \{roberto.piazza, bhavani.shankar, bjorn.ottersten\}@uni.lu \\ ${ }^{\dagger}$ Royal Institute of Technology (KTH), Sweden
}

\begin{abstract}
Sharing the on-board high power amplifier among different uplinked carriers (links) is attractive since it provides for economical and sustainable satellite missions. However, the non-linear characteristic of the satellite amplifier introduces intermodulation products leading to Adjacent Channel Interference (ACI), thereby degrading performance, more so for the spectrally efficient modulations. Towards supporting higher order modulations, this work proposes a novel distortion mitigation technique at the transmitter (predistortion) based on orthogonal memory polynomials and highlights its salient features: performance improvement, scalability and low complexity.
\end{abstract}

\section{INTRODUCTION}

The economical and efficient offerings from the terrestrial networks have strongly motivated the satellite community towards devising economical missions and use of waveforms with improved spectrally efficiency. Sharing of satellite resources among multiple services and advanced transceiver techniques are being considered towards this goal. A typical satellite resource sharing scenario is the joint amplification of multiple channels/ carriers using a single wideband HPA (High Power Amplifier) onboard a transparent satellite instead of dedicated HPAs per channel. This allows for a relaxation of the payload-critical requirements on mass/ power. However, signal amplification, a key onboard operation, is inherently non-linear due to the HPA characteristics and hence an efficient power amplification introduces distortions limiting the use of spectrally efficient modulation schemes [1]. Typically, the combination of HPA non-linearity with the onboard channelizing filters, introduces non-linear inter-symbol interference (ISI) [1]. Multiple carrier power amplification introduces further impairments in the form of severe non-linear adjacent channel interference $(\mathrm{ACI})$ due to the generated intermodulation terms. Higher order modulations face severe distortion and a high guard-band is usually applied when the amplifier is operated in multicarrier mode. Additionally, the high peak to average power ratios in multicarrier operation leads to increased backoff causing a loss in power amplification efficiency. An improvement in power and spectral efficiencies warrants the development of on-ground mitigation techniques including predistortion (PD) at transmitter and equalization at receiver. This is because on-board processing increases mass/ power consumption and is less amenable to enhancements.

Mitigation techniques based on Volterra series [2], [3] or look-up tables (LUT) [1] have been proposed in satellite literature and the resulting gains are promising [1], [4].
Further, literature on terrestrial systems describes a variety of mitigation algorithms based on memory polynomials [5], orthogonal polynomials [6], [7] and LUT [8] for single carrier operations. However, these methods are not suited for the multicarrier scenario since they do not cater to ACI reduction.

Volterra analysis for non-linear satellite channels is developed for two carriers in [9] and extended to multiple carriers in [10]. Different joint data equalization schemes based on Volterra series are then pursued. However, due to compatibility issues, complexity considerations and access restrictions to data on different carriers, the receiver can demodulate only its intended carrier, thereby ruling out joint equalization. On the contrary, the gateway has access to data on all carriers, allowing the implementation of a joint predistortion technique to pre-compensate for ACI and ISI. A dual carrier channel signal predistortion based on memory polynomials (MP) [5] is provided in [11] for terrestrial application. Multicarrier predistortion for satellite channels has been only addressed in [12] where joint data predistortion based on memory polynomials (MP) is considered.

Orthogonal polynomials were developed to improve the accuracy of kernel estimation in the single carrier case [7]. These polynomials are orthogonal in statistical sense when restricted to the memoryless terms [7]. In this work, we investigate the use of orthogonal memory polynomials for multicarrier predistortion. Towards this end, we devise a systematic approach for generating orthogonal MP exploiting data from all carriers. Our approach ensures the orthogonality of polynomials in the statistical sense [7] without any restrictions. The salient features of the new multicarrier orthogonal MP are highlighted and their implications on system design are described. Of particular interest are reduced complexity and modularity: simpler estimation of polynomial co-efficients reduces complexity and the ability to increase the degree/ memory of the predistorter without altering the already computed coefficients imparts scalability.

The rest of the paper is described as follows: Section II describes the multicarrier scenario with emphasis on data predistortion, Section III introduces the novel predistorter whose performance is then compared in Section IV with reported techniques and some conclusions are drawn in Section V.

Notations : A denotes matrices, b represents column vectors, ${ }^{*},{ }^{T}$ are complex conjugation (hermitian) and transposition operators respectively, $\dagger$ denotes the pseudo inversion and 


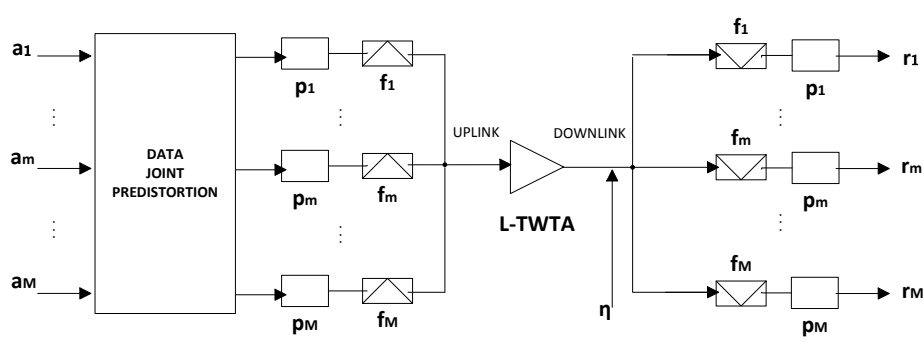

Fig. 1. Multicarrier Channel Model

$\mathbb{E}$ is the ensemble average.

\section{Joint MUlticARrier AmplificAtion}

\section{A. Multicarrier Satellite System}

1) Scenario: The considered multicarrier satellite system involves broadcasting in Ku-band from a geostationary satellite to fixed terminals. A single gateway transmitting independent carriers is assumed and each carrier could correspond to a different service or an application. The user terminal is assumed to be a legacy receiver (e.g. a commercial TV decoder) capable of demodulating and decoding a single carrier, unlike in [9], [10]. This requirement arises from compatibility constraints, complexity considerations and access restrictions. Such single carrier user-terminals cannot compensate for ACI [9], [12]. On the other hand, the predistorter at the gateway is assumed to have information about all the channels; this can be exploited to mitigate ACI.

2) Channel: Figure 1 illustrates a baseband model for the considered system. This model differs from [10] only in the choice of the mitigation technique. The filters, $\left\{p_{i}\right\}$, which add memory effects, represent a cascade of pulse shaping and on-board channelizing filters. TWTAs (Traveling Wave Tube Amplifier) constitute the commercially used on-board HPA; their characteristic is intrinsically non-linear and can be assumed to be frequency independent. Such a memoryless system is characterized by the AM/AM and AM/PM curves [1] and the Saleh model [13] is widely used to parameterize them. To focus on the HPA impairments, we assume an ideal uplink and an AWGN channel for the downlink.

3) Data Predistortion: Unlike in terrestrial scenarios [5], [11], the predistorter and HPA are not co-located due to payload constraints and the need for flexibility. Hence, the predistorter output has to adhere to strict regulations on out-ofband emissions for the uplink. Since a traditional Signal level predistorter [5], [14] causes spectral regrowth, we implement a Data Predistorter (DPD) preceding the pulse shaping filter and operating jointly on multiple carrier data. Clearly, such a DPD does not cause spectral enlargement and is preferred for satellite uplink [1].

\section{B. Data Predistortion Techniques}

Unlike the typical single carrier channels, the considered system is characterized by strong ACI that dominates ISI and the same is well documented in [10], [12]. Central to developing a model based predistortion technique is the ability to parameterize the channel. The channel, essentially a nonlinear system with memory can be completely described using the discrete Volterra series as detailed in [10]. The Volterra analysis then forms the basis for the predistorter design.

1) Volterra DPD: In [10], the derived Volterra series is used towards devising an equalizer. Exploiting the fact that the post-inverse and pre-inverse are ideally the same [15], such an equalizer can also be used as a discrete Volterra multicarrier DPD. In general, the Volterra DPD has infinite order and memory; it is truncated to third or fifth degree for ease of implementation resulting in the output, $y_{m, V}(n)$, with

$$
\begin{aligned}
& y_{m, V}(n)=y_{m, V}^{(1)}(n)+y_{m, V}^{(3)}(n)+y_{m, V}^{(5)}(n) \\
& y_{m}^{(1)}(n)=\sum_{m_{1}=1}^{M_{c}} \sum_{k=-K_{m_{1}}^{(1)}}^{K_{m_{1}}^{(1)}} g_{m_{1}, m}^{(1)}(k) a_{m_{1}}(n-k)
\end{aligned}
$$

where $y_{m}^{(p)}(n)$ denotes the $p$ th degree Volterra term (the general expression is given in (2), top of the next page). $M_{c}$ is the number of carriers, $K_{m}^{(p)}$ denotes memory for the $p$ th degree term for carrier $m,\left\{g_{*}^{(p)}(\cdot)\right\}$ are the $p$ th degree Volterra kernel coefficients, $a_{m}(n)$ and $\eta_{m}(n)$, respectively, are the data symbols and receiver noise on carrier $m$ at instance $n$. The simplified DPD of (2) uses only those nonlinear terms that produce in-band ISI and ACI. In this paper, we define $\Omega_{m, D}$ as the set of carriers $\left(m_{1}, \ldots, m_{D}\right)$ causing in-band distortions to carrier $m$ due to the non-linear terms of degree $D$. The sets $\Omega_{m, D}$ for many significant scenarios are summarized in [10]. Volterra predistortion of degree $D$ requires $O\left(K^{D+1}\right), K=\max _{m}\left\{K_{m}^{(D)}\right\}$.

2) Memory Polynomial DPD (MP-DPD): Volterra DPD predistorter is highly complex due to a large number of cross memory terms involved (even after confining the terms to $\Omega_{*}$ ). This invariably leads to estimation inaccuracies of $\left\{g_{*}^{(p)}(\cdot)\right\}$ based on training. On the other hand, low complexity multicarrier MP-DPD has been derived in [12] developing on their application to single carrier scenarios. The output of a MPDPD, $y_{m}(\cdot)$, takes the form,

$$
y_{m}(n)=\sum_{w=1}^{W} \sum_{k=-K_{w}}^{K_{w}} h_{w, m}(k) \Phi_{m, w, k}(\mathbf{a}(n))
$$

where $\left\{\Phi_{m, w, k}(\cdot)\right\}$ constitutes the standard multicarrier memory polynomial bases described in [12], $\left\{h_{w, m}(\cdot)\right\}$ are the kernel coefficients and $\mathbf{a}(n)=\left[a_{1}(n), \ldots, a_{M_{c}}(n)\right]$. Table I details $\Phi_{m, w, k}(\cdot)$ for cross terms up to the fifth degree and memory depth $2 K_{w}$. Complexity of MP-DPD of degree $D$ is $O(K D), K=\max _{w}\left\{K_{w}\right\}$ compared to $O\left(K^{D+1}\right)$ of Volterra DPD. We now describe a novel DPD based on multicarrier Orthogonal memory polynomials that allows for faster kernel estimation.

\section{Orthogonal Memory Polynomials Based Data PREDISTORTER}

Orthogonal polynomials were introduced in [7] as a signal predistortion mechanism to reduce inaccuracies in estimation 


$$
y_{m, V}^{(p)}(n)=\sum_{\left(m_{1}, \ldots m_{p}\right) \in \Omega_{m, p}} \sum_{k_{1}=-K_{m_{1}}^{(p)}}^{K_{m_{1}}^{(p)}} \ldots \sum_{k_{p}=-K_{m_{p}}^{(p)}}^{K_{m_{p}}^{(p)}} g_{m_{1}, m_{2}, \ldots, m_{p}, m}\left(\left\{k_{l}\right\}_{l=1}^{p}\right) \prod_{s=1}^{\frac{p+1}{2}} a_{m_{s}}\left(n-k_{s}\right) \prod_{s=\frac{p+3}{2}}^{p} a_{m_{s}}^{*}\left(n-k_{s}\right)
$$

TABLE I

Standard Multicarrier Memory Polynomial Basis

\begin{tabular}{|c|c|}
\hline & $\Phi_{m, w, k}(\mathbf{a}(n)), \quad k \in\left[-K_{w}, K_{w}\right]$ \\
\hline Linear terms & $a_{w}(n-k)$ \\
$3^{\text {rd }}$ degree terms & $\left\{\begin{array}{c}a_{m_{3}}^{*}(n-k) \prod_{i=1}^{2} a_{m_{i}}(n-k) \\
\left(m_{1}, m_{2}, m_{3}\right) \in \Omega_{m, 3}\end{array}\right.$ \\
$5^{\text {th } \text { degree terms }}$ & $\left\{\begin{array}{l}\prod_{i=1}^{3} a_{m_{i}}(n-k) \prod_{i=4}^{5} a_{m_{i}}^{*}(n-k) \\
\left(m_{1}, m_{2}, m_{3}, m_{4}, m_{5}\right) \in \Omega_{m, 5}\end{array}\right.$ \\
\hline
\end{tabular}

of kernel coefficients and hence improve out-of-band emissions. While out-of-band emission is not an issue here, as will be shown in the sequel, these polynomials exhibit interesting properties that warrant their use in the multicarrier scenario.

\section{A. Basis representation of the predistortion function}

Let $\left\{\psi_{m, w, k}(\cdot)\right\}, m \in\left[1, M_{c}\right], w \in[1, W], k \in\left[-K_{w}, K_{w}\right]$ denote the set of basis functions and denote, $\psi_{m, i, k}(\mathbf{a}(n))=$ $\psi_{m, i, k}\left(a_{1}(n-k), \ldots, a_{M_{c}}(n-k)\right)$. Motivated by their form in [7], a novel multicarrier data predistorter based on orthogonal polynomials is defined similar to (3) as,

$$
y_{m}(n)=\sum_{w=1}^{W} \sum_{k=-K_{w}}^{K_{w}} h_{w, m}(k) \psi_{m, w, k}(\mathbf{a}(n)) \forall m,
$$

where $y_{m}(n)$ is the predistorter output for the $m$ th carrier at the $n$th instance and $\left\{h_{w, m}(k)\right\}$ are the kernel coefficients. Note that predistorted output for $m$ th carrier depends on symbols from other carriers and utilizes a memory depth of $2 K_{w}$ for each polynomial term $w$. Similar to [7], these basis functions are constrained to satisfy statistical orthonormality as defined by below,

$$
\begin{gathered}
<\psi_{m, i, k}(\mathbf{r}), \psi_{m, j, l}(\mathbf{r})>=\mathbb{E}\left\{\psi_{m, i, k}(\mathbf{r}(n))\left[\psi_{m, j, l}(\mathbf{r}(n))\right]^{*}\right\} \\
<\psi_{m, i, k}(\mathbf{r}), \psi_{m, j, l}(\mathbf{r})>=\left\{\begin{array}{cc}
0 & \forall j \neq i, k \neq l \\
1 & i=j, k=l
\end{array}\right.
\end{gathered}
$$

where $r_{m}(n)$ is the received signal on carrier $m$ at instance $n, \psi_{m, i, k}(\mathbf{r}(n))=\psi_{m, i, k}\left(r_{1}(n-k), \ldots, r_{M_{c}}(n-k)\right)$ and the averaging is performed over the statistics of $\left\{r_{m}(\cdot)\right\}$.

\section{B. Basis Orthogonalization}

We now proceed with the construction of $\left\{\psi_{m, w, k}(\cdot)\right\}$ satisfying (5). The approach is two fold : (1) to choose a set of standard bases functions and (2) obtain an orthonormal set from these bases functions. With regards to the first requirement, motivated by [5], [12], we choose the standard bases functions corresponding to the multicarrier MP as described in Table I. With the standard basis defined, we use the Modified Gram Schmidt method for orthogonalization [16]. For the ease of comprehension, we present the procedure for $K_{w}=0, \forall w$ and the same can be extended to any $K_{w}$. With $K_{w}=0$, dropping subscript $k$ and the input arguments of the bases for simplicity, the standard iterative Gram Schmidt [16] process for the generation of an orthonormal basis $\psi_{m, w}$ from a general basis $\Phi_{m, w}$ is defined in (6),

$$
\begin{aligned}
& \Psi_{m, w}=\Phi_{m, w}-\sum_{z=1}^{w-1} \frac{<\Phi_{m, w}, \Psi_{m, z}>}{<\Psi_{m, z}, \Psi_{m, z}>} \Psi_{m, z}, \\
& \psi_{m, w}=\frac{\Psi_{m, w}}{\left|\Psi_{m, w}\right|} .
\end{aligned}
$$

However, this process suffers from numerical instability and the Modified Gram Schmidt method overcomes this problem by computing each basis $\Psi_{m, w}$ as a sequence of recurrent inner products rather than a summation of inner products,

$$
\begin{aligned}
\Psi_{m, w}^{(1)} & =\Phi_{m, w}-\frac{<\Phi_{m, w}, \Psi_{m, 1}>}{<\Psi_{m, 1}, \Psi_{m, 1}>} \Psi_{m, 1} \\
\Psi_{m, w}^{(2)} & =\Psi_{m, w}^{(1)}-\frac{<\Psi_{m, w}^{(1)}, \Psi_{m, 2}>}{<\Psi_{m, 2}, \Psi_{m, 2}>} \Psi_{m, 2} \\
\vdots & \\
\Psi_{m, w} & =\Psi_{m, w}^{(w-2)}-\frac{<\Psi_{m, w}^{(w-2)}, \Psi_{m, w-1}>}{<\Psi_{m, w-1}, \Psi_{m, w-1}>} \Psi_{m, w-1} .
\end{aligned}
$$

In effect, the Modified Gram Schmidt procedure returns a vector of coefficients $c_{w, l}$ such that each orthonormal basis $\psi_{m, w}$, can be written as a linear combination of the standard basis functions: $\psi_{m, w}=\sum_{l=1}^{w} c_{w, l} \Phi_{m, l}$.

Having defined the functional form of the bases and an orthogonalization procedure, it remains to compute the various correlation coefficients, denoted using $\langle\cdot, \cdot\rangle$ in (7), and estimate the kernel coefficients towards implementing (4).

\section{Computing the Correlation Coefficients}

We now compute the correlation coefficients so that the bases satisfy (5). In this work, no a priori assumption is made on the distribution of the received symbols. Training symbols are used and channel statistics are extracted from the corresponding noisy received data. We approximate the ensemble average by time average based on $r_{m}(n)$ as,

$$
<\Phi_{m, i, k}(\mathbf{r}), \Psi_{m, j, l}(\mathbf{r})>\approx \frac{\sum_{n=1}^{N_{t r}} \Phi_{m, i, k}(\mathbf{r}(n))\left[\Psi_{m, j, l}(\mathbf{r}(n)]^{*}\right.}{N_{t r}}
$$

Remark 1: In [7], a closed-form expression for polynomials is obtained for a single carrier and for a specific distribution of $\left\{r_{1}(n)\right\}$. Further, the orthogonality is satisfied for the memoryless terms. However, the construction provided above can be applied regardless of the polynomial degree, distribution of received symbols and channel characteristics. Further, the proposed construction imposes orthogonality both on the polynomial terms $w$ as well as on the memory $k$ of the orthogonal MP (kindly refer to (4)). 


\section{Kernel Estimation}

It now remains to obtain the kernel coefficients $\left\{h_{w, m}(k)\right\}$. By virtue of being used for DPD, $\left\{h_{w, m}(k)\right\}$ model the inverse of the channel function. Thus $\left\{h_{w, m}(k)\right\}$ can be estimated using the received symbols by modeling predistortion as a post-inverse [15]. Typically, $\left\{h_{w, m}(k)\right\}$ are computed prior to launch by measuring the HPA characteristics and simulating the satellite transmission [1]. However, aging and temperature variations (diurnal variations as the satellite moves back and forth from the shadow of earth) changes the channel characteristics. These motivate a periodic, training based, estimation of $\left\{h_{w, m}(k)\right\}$ when the satellite is in operation using operator owned dedicated reference terminals capable of multicarrier demodulation. These terminals compute $\left\{h_{w, m}(k)\right\}$ and feed them back to the gateway.

Recalling the definition of $r_{i}(n), a_{i}(m)$ from earlier Sections, the post-inverse takes a form similar to (4) with,

$$
a_{m}(n)=\sum_{w=1}^{W} \sum_{k=-K_{w}}^{K_{w}} h_{w, m}(k) \psi_{m, w, k}(\mathbf{r}(n))+\epsilon_{m}(n)
$$

where $\mathbf{r}(n)=\left[r_{1}(n), \ldots, r_{M_{c}}(n)\right]$ and $\epsilon_{m}(n)$ is the modeling error. Stacking $N_{t r}$ number of training symbols $\left\{a_{m}(n)\right\}$ into a vector $\mathbf{b}_{m}$ and letting $\mathbf{h}_{m}=\left[h_{1, m}\left(-K_{1}\right)\right.$, $\left.\ldots, h_{1, m}\left(K_{1}\right), h_{2, m}\left(-K_{2}\right), \ldots, h_{W, m}\left(K_{W}\right)\right]^{T}$ we can write (9) as $\mathbf{b}_{m}=\mathbf{A}_{m} \mathbf{h}_{m}+\mathbf{e}_{m}$. Here, $\mathbf{e}_{m}$ is the stacked error vector, and $\mathbf{A}_{m}$ is a matrix whose $p$ th row corresponds to the evaluation of $\left\{\psi_{m, w, k}()\right\}$ for $p$ th received symbol. Based on this relation, $\left\{h_{w, m}(k)\right\}$ are obtained by minimizing, $\left[\mathbf{A}_{m} \mathbf{h}_{m}-\mathbf{b}_{m}\right]^{*}\left[\mathbf{A}_{m} \mathbf{h}_{m}-\mathbf{b}_{m}\right]$, as,

$$
\mathbf{h}_{m}=\mathbf{A}_{m}^{\dagger} \mathbf{b}_{m}, m \in\left[1, M_{c}\right] .
$$

Equation (10) involves a complexity of $O\left(N_{t r} K^{2} W^{2}\right), K=$ $\max _{w} K_{w}$ and can be susceptible to ill conditioning of $\mathbf{A}_{m}$. The reduction in complexity estimation is described next.

\section{E. Properties of Orthogonal Bases}

1) Low Complexity Kernel Estimation: The lth column of $\mathbf{A}_{m}$ corresponds to the evaluation of $\psi_{m, w_{0}, k_{0}}(\cdot)$ (for some $\left.w_{0}, k_{0}\right)$ on the $N_{t r}$ symbols. From (8), it can be therefore deduced that the columns of $\mathbf{A}_{m}$ are orthonormal. In fact, $\mathbf{A}_{m}$ is the orthogonal component (computed using QR decomposition) of the regression matrix corresponding from MP. Due to orthonormality, (10) can be simplified as,

$$
\mathbf{h}_{m}=\mathbf{A}_{m}^{*} \mathbf{b}_{m}
$$

Equation (11) shows that estimation procedure is simplified to a large extent by use of orthogonal MP with the complexity being only $O\left(N_{t r} K W\right)$. This simplification leads to cheaper reference terminals.

2) Modularity: An important manifestation of (11) is modularity; additional basis functions (in terms of degree and/ or memory) could be included and the kernel coefficients corresponding to these new functions can be found without altering those estimated already. This arises from the fact that adding a new basis appends a new column to $\mathbf{A}_{m}$.
Now, referring to (11), the corresponding kernel coefficient can simply be found as the inner product of new column of $\mathbf{A}_{m}$ and $\mathbf{b}_{m}$. When reference receive terminals are used, this scalability allows for a reduction in the amount of feedback. Similarly, the number of basis functions can be reduced by merely nulling the appropriate kernel coefficients. This feature provides for a control of received signal quality by a simple alteration of the memory or degree of the predistorter.

\section{Simulation Results}

\section{A. Performance Metric}

We now illustrate the performance of the proposed predistorter. The traditional measure of performance for non-linear channels is the Total Degradation (TD) [1]. In this paper, we instead use the Signal to Interference plus Noise Ratio (SINR) as the performance metric since it (i) does not involve bit error rate evaluations and is faster to compute and (ii) is compatible to TD in behavior. In particular, the SINR for carrier $m$ would be $\rho_{m}=\frac{\mathbb{E}\left(\left|a_{m}(n)\right|^{2}\right)}{\mathbb{E}\left(\left|\alpha_{m} r_{m}(n)-a_{m}(n)\right|^{2}\right)}$, where $\alpha_{m}>0$ effects an unit power normalization to the desired signal at the receiver (depends on the set amplification level). When the Input Backoff (IBO) increases, the non-linear interference reduces but the signal strength after amplification also reduces thereby increasing the relative noise level $\left(\alpha_{m}>1\right)$. Reducing IBO increases signal power compared to noise, but the non-linear interference also increases. These effects are well captured in the denominator of $\rho_{m}$. Hence, similar to TD, there exists an optimum IBO (or equivalently Output Backoff, OBO) at which $\rho_{m}$ is maximized.

\section{B. Set-Up}

Simulations have been carried out with two and three carriers $\left(M_{c}=2,3\right)$ per HPA. Saleh model [13] is used to obtain

TABLE II

SiMULATION PARAMETERS

\begin{tabular}{|c|c|}
\hline Symbol rates, $R_{s}$ in MBaud & $8\left(M_{c}=3\right), 12\left(M_{c}=2\right)$ \\
\hline Carrier frequency spacing, $\triangle f$ & $1.25 R_{s}$ \\
\hline$\{$ DPD degree, DPD memory $\}$ & $\left\{5\right.$ (Table I), $K_{w}=1, \forall w$ in eq. (4) $\}$ \\
\hline Number of training symbols & 3200 (A DVBS2 short frame) \\
\hline Modulation & 32 APSK \\
\hline Filters, $\left\{p_{i}\right\}$ & Root Raised Cosine, roll-off 0.25 \\
\hline Simulation Oversampling & $38\left(M_{c}=3\right), 25\left(M_{c}=2\right)$ \\
\hline$E_{s} / N_{o}$ & $20 \mathrm{~dB}$ \\
\hline
\end{tabular}

the HPA characteristic with the same parameters as in [10]. As in [10], the pilots are drawn from 32 APSK constellation (target modulation). For comparing with the proposed scheme (depicted as Orth. MP-DPD), a MP-DPD of Section II-B2 is designed [12]. For the current scenario, Volterra DPD is omitted due to its exponential complexity (kindly refer Section II-B2). Further the benchmark cases of No DPD (without any HPA non-linearity compensation at the receiver) and an ideal linear amplifier with normalized gain (denoted as AWGN channel) are also simulated. 


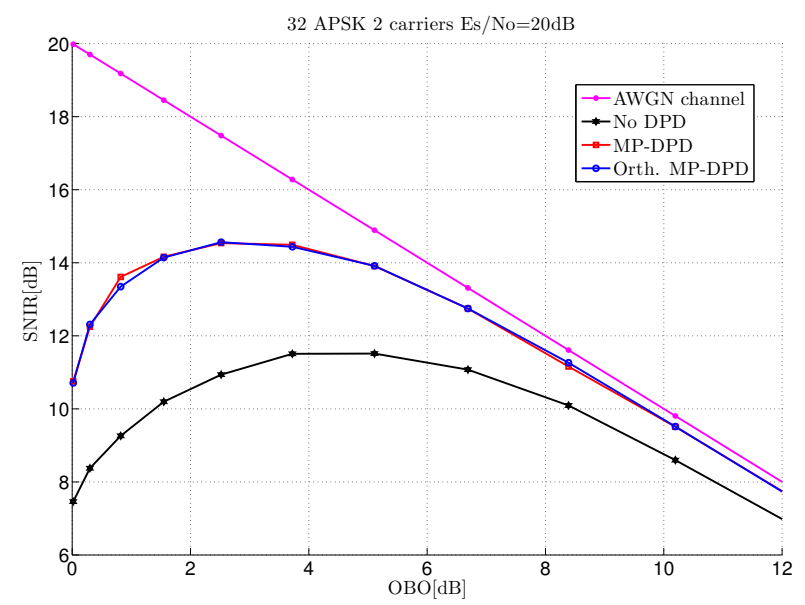

Fig. 2. SINR performance of different DPDs : $M_{c}=2$

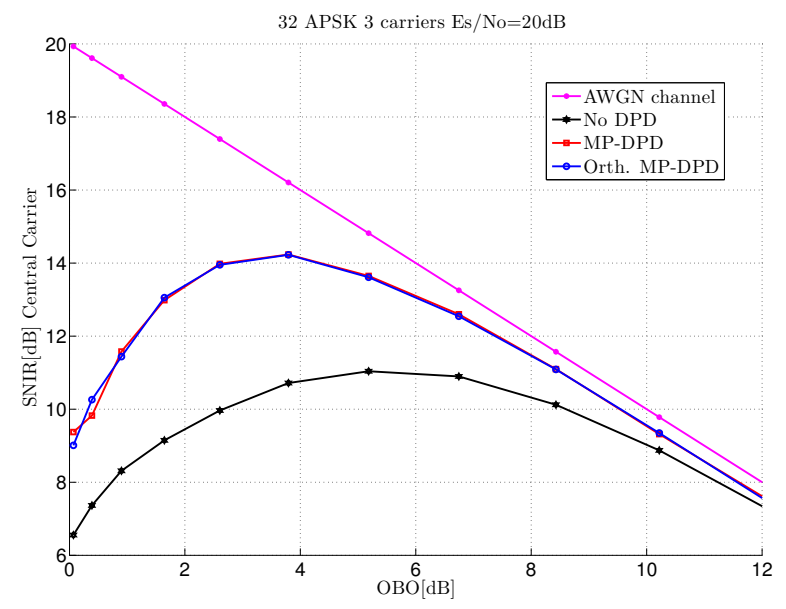

Fig. 3. SINR performance of different DPDs : $M_{c}=3$, (Central Carrier)

\section{Results}

When $M_{c}=2$, because of symmetry, both carriers have the same SINR and hence only one is depicted in Fig. 2. For $M_{c}=3$, performance of the central carrier is impacted by strong ACI from the two external carriers. Hence the SINR of central carrier is shown in Fig. 3, while the performance of the external carriers are similar to Fig 2. Performance of the designed DPD is similar to MP-DPD for an identical order and memory depth, while providing the benefits outlined in Section III-E. Comparing with the No DPD case, the use of predistortion effectively compensates the non-linear effects of the channel providing about $3 \mathrm{~dB}$ of gain in the region of high power efficiency $(\mathrm{OBO} \approx 2.5 \mathrm{~dB}$ ). Further, this SINR gain can also be translated into a power efficiency improvement: for a target SINR, the OBO can be significantly reduced by applying DPD. Comparing Fig. 2 and Fig. 3, we notice that a higher number of carriers introduces greater ACI, thereby reducing the absolute SINR. As a consequence of this, increasing $M_{c}$ gradually moves the optimum $\mathrm{OBO}$, corresponding to the maximum SINR, towards the linear region of the amplifier. As expected, the DPD performance approaches the linear amplification case for high OBO (small difference being due to the modeling/ estimation errors). The No DPD case asymptotically (in $\mathrm{OBO}$ ) reaches the performance of the AWGN case, mainly due to the slow decay of the HPA phase with OBO [13].

\section{CONClusion}

The paper presents a novel framework for generating and applying orthogonal memory polynomials as a predistortion technique when multiple carriers are amplified by a single HPA. This technique provides for a mitigation of ACI and ISI thereby improving received SINR and power efficiency. Exploiting orthogonality reduces the complexity while resulting in a modular (scalable) implementation. These properties provide for a favorable comparison of the proposed technique with prior-art strongly motivating its use in evolving systems.

\section{REFERENCES}

[1] E. Casini, R. De Gaudenzi, and A. Ginesi, "DVB- S2 modem algorithms design and performance over typical satellite channels," Intern. J.on Satellite Commun. and Networking, vol. 22, pp. 281-318, 2004.

[2] S. Benedetto and E. Biglieri, "Nonlinear equalization of digital satellite channels," IEEE J. Sel. Areas Commun., vol. 1, pp. 57 -62, Jan. 1983.

[3] G. Colavolpe and A. Piemontese, "Novel SISO detection algorithms for nonlinear satellite channels," in Proc. IEEE Global Telecommun. Conf.e, Houston, USA, Dec. 2011.

[4] L. Giugno, M. Luise, and V. Lottici, "Adaptive pre and postcompensation of nonlinear distortions for high-level data modulations," IEEE Trans. Wireless Commun., vol. 3, pp. 1490 -1495, 2004.

[5] L. Ding, G. T. Zhou, D. R. Morgan, Z. Ma, J. S. Kenney, J. Kim, and C. R. Giardina, "A robust digital baseband predistorter constructed using memory polynomials," IEEE Trans. Commun., vol. 52, no. 1, pp. 159 165, Jan. 2004.

[6] D. Ronnow and M. Isaksson, "Digital predistortion of radio frequency power amplifiers using kautz-volterra model," Electronics Letters, vol. 42, no. 13, pp. 780 - 782, June 2006.

[7] R. Raich, H. Qian, and G. Zhou, "Orthogonal polynomials for power amplifier modeling and predistorter design," IEEE Trans. Veh. Technol., vol. 53, no. 5, pp. 1468 - 1479, Sept. 2004.

[8] G. Karam and H. Sari, "A data predistortion technique with memory for QAM radio systems," IEEE Trans. Commun., vol. 39, no. 2, pp. 336 -344 , feb 1991.

[9] B. F. Beidas and R. Seshadri, "Analysis and compensation for nonlinear interference of two high-order modulation carriers over satellite link," IEEE Trans. Commun., vol. 58, no. 6, pp. 1824 -1833, June 2010.

[10] B. F. Beidas, "Intermodulation distortion in multicarrier satellite systems: Analysis and turbo volterra equalization," IEEE Trans. Commun., vol. 59, no. 6, pp. $1580-1590$, June 2011.

[11] S. Bassam, M. Helaoui, and F. Ghannouchi, "2-D digital predistortion (2-D-DPD) architecture for concurrent dual-band transmitters," Microw. Theory and Techniques, IEEE Trans. on, vol. 59, no. 10, pp. 2547 -2553, oct. 2011.

[12] R. Piazza, E. Zenteno, and e. al, "Multicarrier digital predistortion/ equalization techniques for non-linear satellite channels," in Proc. 30th AIAA Intern.Commun. Satellite Syst. Conference (ICSSC), Ottawa, Canada, Sep. 2012. [Online]. Available: http://publications.uni.lu/record/10106/files/

[13] A. Saleh, "Frequency-independent and frequency-dependent nonlinear models of twt amplifiers," IEEE Trans. Commun., vol. COM-29, no. 11, pp. 1715-1720, November 1981.

[14] G. E. Corazza, Digital Satellite Communications, Chapter 8. Springer, 2007.

[15] M. Schetzen, The Volterra and Wiener Theories of Nonlinear Systems. John Wiley \& Sons, Apr. 1980. [Online]. Available: http://www.worldcat.org/isbn/0471044555

[16] A. Bjorck, "Solving linear least-squares problems by gram-schmidt orthogonalization," BIT Numerical Mathematics, vol. 7, pp. 1-21, 1967. 